\title{
Development of a Continuous Blood Pressure Measurement and Cardiovascular Multi-Indicator Platform for Asian Populations by Using a Back Propagation Neural Network and Dual Photoplethysmography Sensor Signal Acquisition Technology
}

\author{
Chih-Ta Yen ${ }^{1}{ }^{1}{ }^{1}$ Sheng-Nan Chang, ${ }^{2}$ and Cheng-Yang Cai ${ }^{3}$ \\ ${ }^{1}$ Department of Electrical Engineering, National Taiwan Ocean University, Keelung City 202301, Taiwan \\ ${ }^{2}$ Division of Cardiology, Department of Internal Medicine, National Taiwan University, Yun-Lin Branch, Dou-Liu City 640, Taiwan \\ ${ }^{3}$ Department of Electrical Engineering, National Formosa University, Yunlin County 632, Taiwan \\ Correspondence should be addressed to Chih-Ta Yen; chihtayen@gmail.com
}

Received 25 December 2020; Revised 1 February 2021; Accepted 30 April 2021; Published 29 May 2021

Academic Editor: Victor M. Castano

Copyright (c) 2021 Chih-Ta Yen et al. This is an open access article distributed under the Creative Commons Attribution License, which permits unrestricted use, distribution, and reproduction in any medium, provided the original work is properly cited.

\begin{abstract}
This study proposed a measurement platform for continuous blood pressure estimation based on dual photoplethysmography (PPG) sensors and a back propagation neural network (BPNN) that can be used for continuous and rapid measurement of blood pressure and analysis of cardiovascular-related indicators. The proposed platform measured the signal changes in PPG and converted them into physiological indicators, such as pulse transit time (PTT), pulse wave velocity (PWV), perfusion index (PI), heart rate (HR), and pulse wave analysis (PWA); these indicators were then fed into the BPNN to calculate blood pressure. The hardware of the experiment comprised 2 PPG components (i.e., Raspberry Pi 3 Model B and analog-to-digital converter [MCP3008]), which were connected using a serial peripheral interface. The BPNN algorithm converted the stable dual PPG signals acquired from the strictly standardized experimental process into various physiological indicators as input parameters and finally obtained the systolic blood pressure (SBP) and diastolic blood pressure (DBP). To increase the robustness of the BPNN model, this study input data of 100 Asian participants into the training database, including those with and without cardiovascular disease, each with a proportion of approximately $50 \%$. The experimental results revealed that the mean and standard deviation of SBP were $2.23 \pm 2.24 \mathrm{mmHg}$, with a mean squared error of $3.15 \mathrm{mmHg}$. The mean and standard deviation of DBP was $3.5 \pm 3.53 \mathrm{mmHg}$, with a mean squared error of $4.96 \mathrm{mmHg}$. The proposed real-time blood pressure measurement system exhibited a mean accuracy of $98.22 \%$ and $95.58 \%$ for SBP and DBP, respectively.
\end{abstract}

\section{Introduction}

Hypertension remains a major factor in cardiovascular disease in Taiwan, and it is also a considerable global health concern. Hypertension is a crucial indicator of whether the human heart is functioning normally. The related treatment process is long and challenging, and people still have extremely low awareness of hypertension. Research has revealed that in those aged between 19 and 44 years, the prevalence of prehypertension is higher than the proportion of patients with hypertension. The age trend is also decreasing, suggesting that cardiovascular disease is no longer a concern only for older adults. Factors such as family medical history, eating habits, living habits, work stress, fatigue, temperature, and aging are all closely related to cardiovascular disease.

Research on photoplethysmography (PPG) is increasing, and numerous scholars have discussed its measurement methods, signal processing, algorithm applications, measurement applications, hardware improvement and innovation, and system construction. As early as 1937, Hertzman and Spielman [1] proposed the concept of PPG by using optical measurement of blood volume changes. Burton [2] observed strong correlations among systolic blood pressure (SBP) of the finger, pulse rate, and the sympathetic nervous system. 
Mendelson and Ochs [3] developed a sensor for reflectance pulse oximetry to evaluate a prototype skin-reflectance pulse oximeter in humans. In 1994, Nitzan [4] used PPG to produce two parameters, namely, amplitude modulation (AM) and baseline, and proved that the PPG signal and heart rate (HR) variability have similar spectral sections. In addition, Task Force [5] and Nakajima [6] have both confirmed the correlation between PPG signal, and HR. Rhee et al. [7] proposed a finger-ring plethysmographic sensor, indicating that PPG can continuously monitor arterial pulsation without causing harm. Numerical simulations and experiments were conducted to verify and evaluate the aforementioned model. In the same year, Chan et al. [8] proposed a noninvasive and cuffless measurements of blood pressure for telemedicine. This technology used the time interval between electrocardiography (ECG) and PPG to predict the systolic, diastolic, and mean blood pressure on the basis of the pulse transit time (PTT) technology, and it applied the wireless application protocol to display information on portable wireless devices Moreover, Murthy et al. [9] analyzed the spectrum of PPG signals in cardiovascular patients; they noted that PPG can be used as an alternative diagnostic tool for studying the cardiovascular system, particularly HR variability. Teng and Zhang [10] confirmed the feasibility of continuous and noninvasive estimation of arterial pressure by using PPG. The aforementioned works have confirmed the application of PPG for measuring and analyzing health conditions and characteristics as feasible; they also revealed that the PPG sensor can effectively reflect the relationship between the heart and the blood vessel, leading to its extensive use.

Various methods for measuring blood pressure-related physiological signals have been proposed. In signal processing, biological signals must be converted to electric potentials before the signals can be processed by computers or control devices. The biological signal is generally weak and accompanied by a range of noise; hence, the signal must be amplified through an amplifier and the noise filtered out. Subsequently, samples are taken through the alternating current $(\mathrm{AC}) /$ direct current (DC) converter; then, the analog signal is converted to a digital signal; finally, the digital signal is input into a computer for analysis. The biological signal mainly includes biomechanical and biooptical signals. Of these two, biomechanical signals are the more widely used in blood pressure measurement, as is the case in ECG and pulse sensors. Biooptical signals are increasingly being used to measure blood pressure, and the most representative measurement tool is PPG. Many related studies have also indicated that multiwavelength PPG can be used for cuffless blood pressure measurement [11-13].

Various methods related to measuring the correlation between blood and optics currently apply PPG for estimating blood pressure. They can generally be classified into PTT, pulse wave velocity (PWV), and pulse wave analysis (PWA). PWV is inversely correlated with PTT. Although the methods differ, the three variables are closely related [14]. However, in recent years, some scholars have proposed perfusion index (PI), which is defined as the ability of the cardiovascular system to provide sufficient blood for normal functioning and metabolism of body tissues. PI is also an essential metric for judging the condition of the cardiovascular system [15].

PTT represents the time interval of the arterial pulse wave from the aortic valve to a peripheral artery; PTT is said to be inversely proportional to the change in blood pressure [16], and it can be used for noninvasive continuous blood pressure estimation. PTT is defined as the time between two specific parts of the PPG signal waveform. Both events occur at each heartbeat and can be easily detected. The main purpose is to study the relationship between the time course of the heart's electrical activity and cardiovascular activity. In the past few decades, PPG has also been widely applied to estimate blood pressure by using PTT [17-22]. Current clinical best practice involves starting the timing from the apex of the $\mathrm{R}$ wave in the ECG to the starting point of the largest upward branch in the PPG of a certain part of the peripheral artery blood flow. This measurement is now collectively referred to as PTT, which is typically the distance from the heart to the wrist or fingertips. Some scholars have also suggested the use of dual PPG to obtain PTT. This process involves using a peak detection algorithm to process two PPG signals and identify the peaks in each cardiac cycle, measuring the blood transit time from shoulder to finger, and calculating the sample difference between the two peaks [23]. Lin et al. [24] used PTT to estimate blood pressure and calibrated the system by using linear regression with reference to multiple data samples of blood pressure. However, under different conditions, the velocity of transferring energy formed by heart activity to the peripheral blood vessel differs. For example, vasoactive substances and autonomic tone can affect heart and blood vessel activity, thereby affecting blood flow.

For PWV, two PPG signals are used to analyze the PPG signal along the same artery branch at a known distance and set position simultaneously. A specific part of the signal is evaluated to identify the difference in time and distance between the two signals; these values are then used to calculate the PWV. This method, which is used to determine arteriosclerosis, has been extensively studied and proven in internal medicine [25]. In clinical medicine, PWV measurements are also used as a diagnostic parameter of arteriosclerosis. Although PWV has proven a direct correlation between blood pressure and measurement velocity [26, 27], a precise distance between the two sensors is required to accurately calculate blood pressure. However, the sensor may move over time or during the measurement, resulting in inaccurate calculation. Moreover, precise placement of the sensor is required to achieve optimal results.

PWA is used to evaluate the pressure waveform generated by systolic and diastolic flow in the arteries. It can accurately record the peripheral pressure waveform and generate the corresponding central waveform, from which the augmentation index and central pressure can be derived. PWA also includes the relevant characteristics of PTT and PWV. Clinical studies have indicated that PWA is a technology that produces reliable results and can be applied easily. Samria et al. [28] were the first to propose that using PWA to assess stiffness may provide a more satisfactory risk assessment and target treatment for those in greatest need. Blood vessel stiffness may not only be a sign of atherosclerosis but may also be 
involved in the pathogenesis of cardiovascular diseases through diverse mechanisms. Therefore, the use of PWA to assess arterial stiffness may provide a more efficient risk assessment and enable treatment to be targeted to those who need it most. Nevertheless, to enable more accurate judgment and assessment, this technology must acquire a complete PPG waveform without interference. Therefore, Joseph et al. [29] suggested the use of discrete wavelet transform to strengthen the waveform characteristics and completeness by eliminating the damaged PPG signal caused by motion artifacts. In addition, Fischer et al. introduced a new embedded algorithm for real-time pulse waveform segmentation and artifact detection based on PPG time domain analysis to analyze various physiological indicators. Furthermore, Zhang [30] proposed a method on the basis of mean impact value combined with a genetic algorithm-back propagation neural network (BPNN) to improve the analysis accuracy of PPG waveforms for predicting blood pressure; the results of SBP and diastolic blood pressure (DBP) were $4.213 \pm 5.609 \mathrm{mmHg}$ and $3.230 \pm 4.591 \mathrm{mmHg}$, respectively, proving again that the condition of the PPG waveform can affect the accuracy of blood pressure estimation.

\section{Experimental and System Setup}

2.1. Participants. The data source is cooperated with physicians from National Taiwan University Hospital, Yun-Lin Branch, to conduct a clinical trial after being granted approval by the relevant institutional review board. A total of 100 people participated in this study, including 57 outpatients from the cardiovascular disease department and 43 students from the National Formosa University. A large sample was desirable for increasing the number of data in the experimental database and improving the robustness of the BPNN algorithm model when measuring SBP and DBP.

(1) The inclusion criteria for this study were as follows:

(i) Inpatients or outpatients with cardiovascular diseases who routinely undergo general physiological parameter examinations

(ii) Students with normal blood pressure

(iii) Patients over 20 years of age

(2) The exclusion criteria were as follows:

(i) Pregnant women

(ii) Children or minors (under 20 years old)

(iii) People with mental disorders

This study used the dual PPG measurement method to capture waveform signals of the human body. The number of male participants was 87 , and their SBP and DBP ranged from 98 to 179 and 32 to 121 , respectively. The SBP and DBP of the 13 female participants ranged from 99 to 193 and 57 to 102, respectively. The participants' age range, average height, average weight, SBP, and DBP are presented in Tables 1 and 2 .

The experimental location was a quiet empty room next to the physician's office; the experimental environment is depicted in Figure 1. During the experiment, the participants did not have any contact or interaction with the physician. At the beginning of the experiment, the participants were provided with detailed information on the process and purpose of the experiment. The experiment started after patients signed the consent form. The crucial elements of the experiment are as follows:

(1) Informed consent form

(2) Dual PPG system

(3) Raspberry Pi 3

(4) Omron HEM-7070 (Omron Corporation, Kyoto, Japan) electronic blood pressure monitor

(5) Lay participant's hands flat on a table, aligned with the position of the heart

(6) Quiet environment

(7) Basic information of experimental participants

In the experimental process, this study referred to a related study [43] on the influence of office blood pressure measurement on the diagnosis and treatment of hypertension. The study explored the use of noninvasive PPG for measuring human photoelectric signals and obtained relevant waveforms to identify the characteristics related to blood pressure. The experimental measurement process, BPNN training framework, and apparatus setup are illustrated in Figures 2, 3, and 4, respectively. The data of the participants were collected through the following procedure:

(1) Male and female volunteers with and without cardiovascular disease were invited to participate in this study. All participants were informed regarding the research objective and experimental methods, and they subsequently signed an informed consent form

(2) The participants placed both their hands at the same height as the heart and sat quietly (without talking) for $5 \mathrm{~min}$; this enabled the researchers to obtain a stable PPG waveform and blood pressure

(3) The inflatable sleeve (Omron) was wrapped around the left upper arm and compressed once every 2 min for a total of three times. The dual PPG was fixed on the index and middle fingers to measure the PPG signal waveform, and the waveform and conversion parameters were continuously recorded for approximately $5 \mathrm{~min}$. The experiment had a duration of $10 \mathrm{~min}$ per person, and the collected data were used as the training data set of the BPNN 
TABLE 1: Characteristics of male participants.

\begin{tabular}{lc}
\hline Parameters & Daily activity \\
\hline Men & 87 \\
Age range (years) & $20-88$ \\
Height $(\mathrm{cm})$ & $171 \pm 17$ \\
Weight $(\mathrm{kg})$ & $74 \pm 14$ \\
SBP range $(\mathrm{mmHg})$ & $128 \pm 15$ \\
DBP range $(\mathrm{mmHg})$ & $81 \pm 13$ \\
\hline
\end{tabular}

TABLE 2: Characteristics of female participants.

\begin{tabular}{lc}
\hline Parameters & Daily activity \\
\hline Women & 13 \\
Age range (years) & $20-84$ \\
Height $(\mathrm{cm})$ & $155 \pm 8$ \\
Weight $(\mathrm{kg})$ & $57 \pm 8$ \\
SBP range (mmHg) & $132 \pm 25$ \\
DBP range $(\mathrm{mmHg})$ & $79 \pm 9$ \\
\hline
\end{tabular}

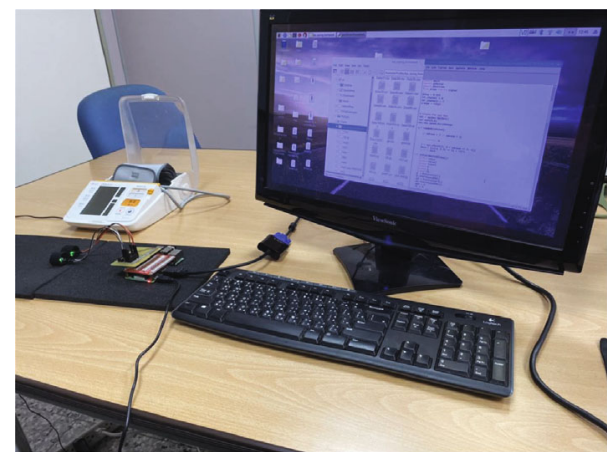

FIGURE 1: Experimental environment and apparatus layout.

(4) The age, height, and weight of the participants were obtained after the experiment

2.2. Apparatus. This study implemented a continuous blood pressure measurement platform. The system included two green-light PPG sensors, a Raspberry Pi 3, an MCP3008, and an Omron blood pressure monitor. The two PPGs were clipped onto the index and middle fingers and were connected to the Raspberry Pi 3 through the serial peripheral interface (SPI). The MCP3008 was used to convert analog signals to digital signals, and Python syntax was used to process PPG signals to obtain signal waveforms and extract the characteristics. Finally, the Omron blood pressure monitor was used to measure blood pressure simultaneously on the same hand. After algorithm analysis and calculation, the researchers conducted conversion to obtain the HR, PTT, PWV, PI, and blood pressure-related data, and the SBP and DBP were calculated. The system structure is illustrated in Figure 5.

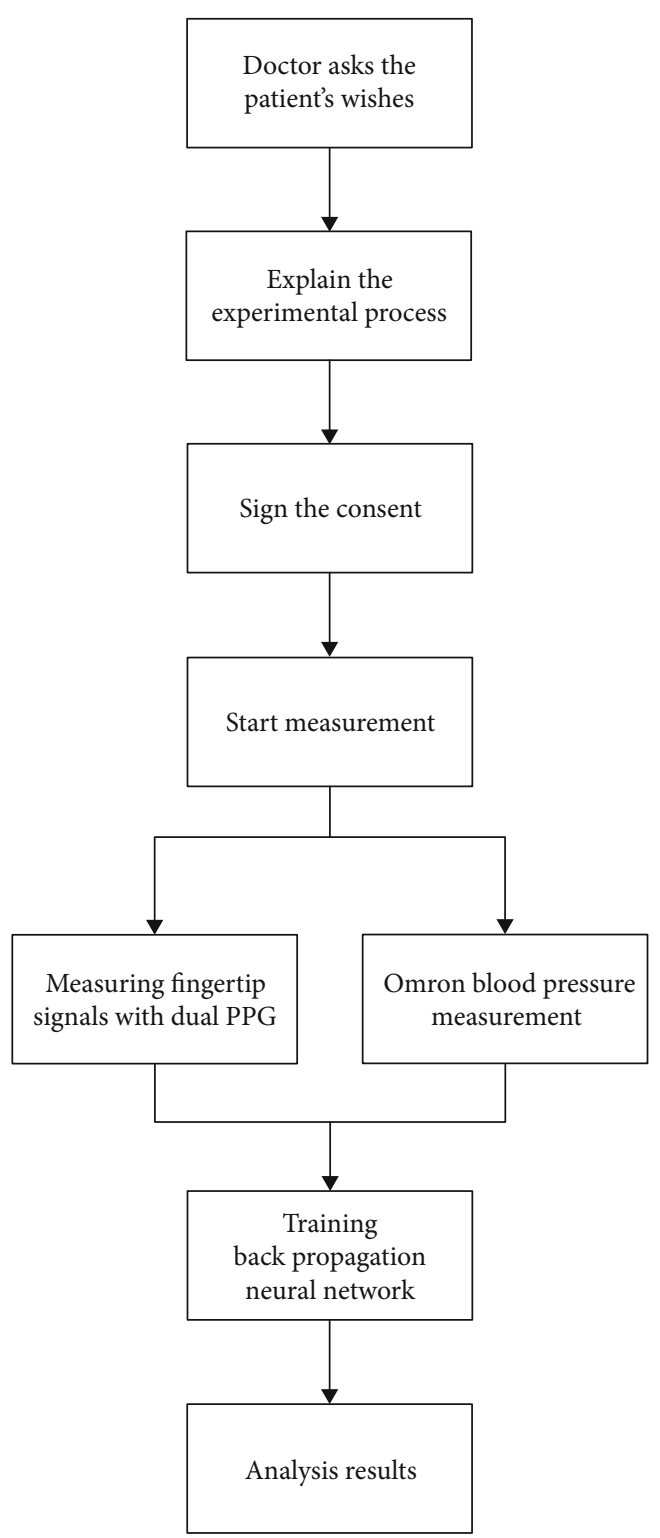

FIgURE 2: Flowchart of the clinical experiment.

2.3. PPG Sensor. PPG is often used in medical research to measure the signals of human subcutaneous tissue and is widely used to monitor the pulse of patients. The key components of a PPG are a light-emitting diode, photodiode, and amplifier. Thus, PPG is a simple and low-cost optical method that can be used to detect changes in blood volume in tissue capillary beds. In addition, the complete waveform composition of the PPG signal is a combination of DC and AC components. The DC component comes from the optical signal reflected or transmitted by tissue and is determined by tissue structure and the volume of venous and arterial blood; the $\mathrm{AC}$ component corresponds to the change in blood volume synchronized with the HR. The DC component indicates small changes in breathing, whereas the AC component changes with the HR and is superimposed on the DC baseline (Figure 6).

In the experiment, two sets of reflective pulse sensors were used, the sampling rate was set to $500 \mathrm{~Hz}$, and the 


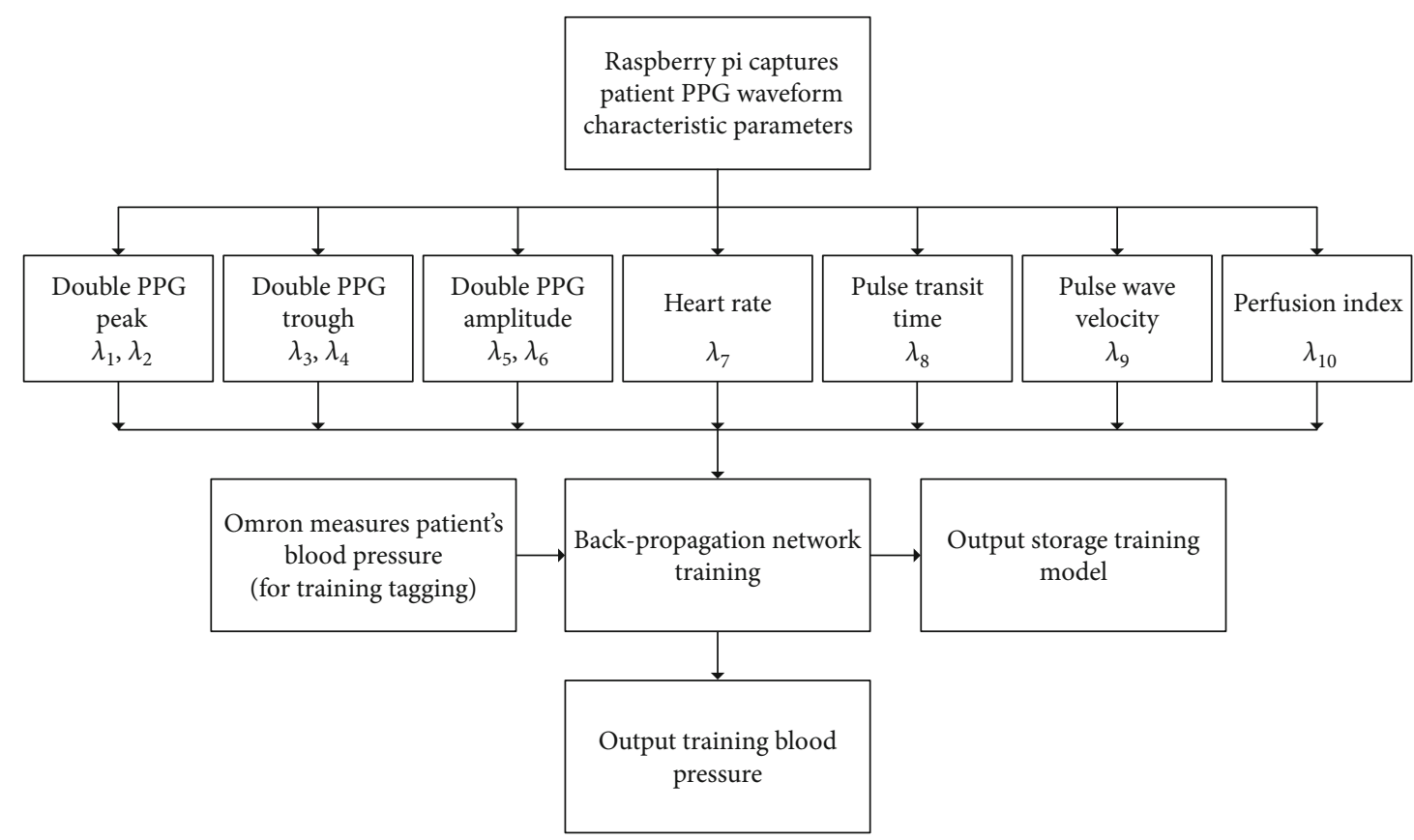

FIGURE 3: Flowchart of the proposed back propagation neural network.

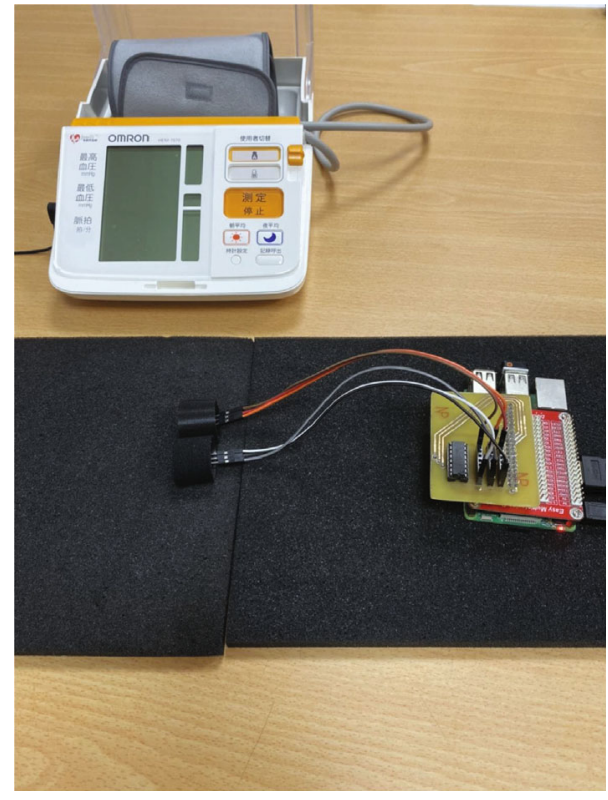

Figure 4: Apparatus setup.

transmitter module used a green light-emitting diode (AM2520ZGC09) with a peak wavelength of $515 \mathrm{~nm}$. The pulse sensors were mainly designed for the multiple demands of discrete backlight and the necessity of maintaining high intensity and wide viewing angle radiation. In addition, the receiver used a miniature surface-mount ambient light photo sensor (APDS-9008) with a sensing peak wavelength of $565 \mathrm{~nm}$. Highly sensitive and similar peak response wavelengths of the transmitter and receiver enable the display of a clearer pulse waveform.

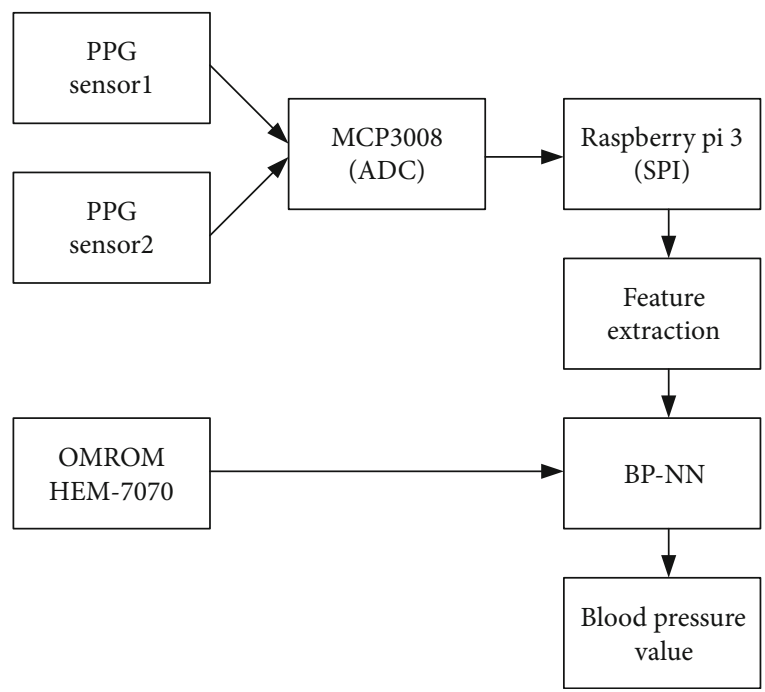

Figure 5: System structure diagram.

The pulse sensor proposed in this study adopted a reflective light sensor, which was tightly attached to the finger with a strap during measurement. The sensor was susceptible to a range of signal interference because the frequency band of the signal was generally between 0.05 and $200 \mathrm{~Hz}$ and the signal amplitude was extremely small (generally at the millivolt). Therefore, a general passive low-pass filter (model of passive RC: R, 100; C, 4.7uF) and MCP6001 were installed at the receiving end. An operational amplifier served as an amplifying filter module, with the purpose of reducing noise, increasing pulse wave amplitude, and amplifying the received signal by 330 times. The DC bias was set to half of the power supply voltage with a voltage divider, and the signal was 


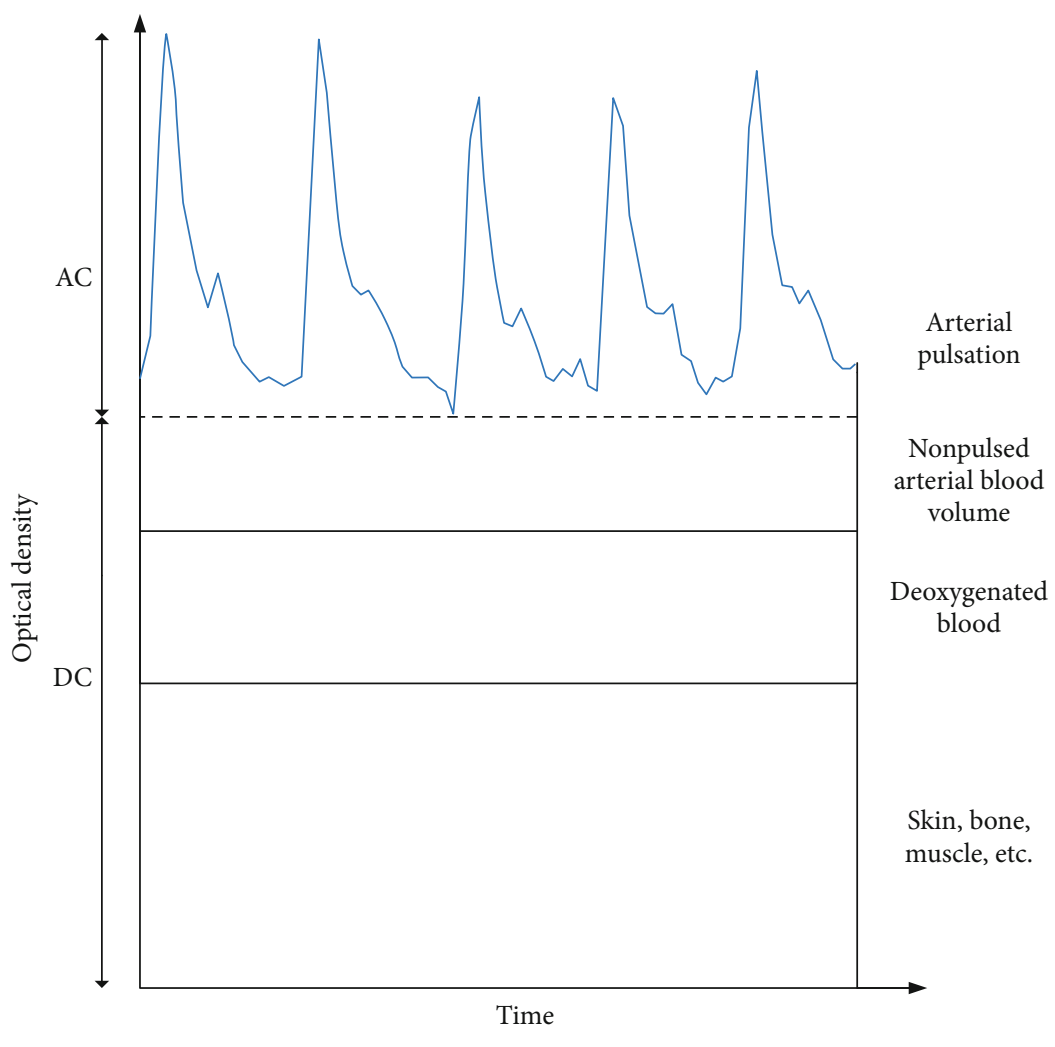

FIgURE 6: PPG signal composed of AC and DC components.

normalized near the reference point to facilitate the collection of the amplified PPG signal waveform by the Raspberry Pi 3. An anti-electric-shock design was also employed to protect the participants. The front and back of the sensor element are depicted in Figure 7.

2.4. Hardware System Design and Connection Method. This experimental system connected the Raspberry Pi 3, MCP3008, and PPG sensor through the SPI. Further details are as follows:

(1) Digital Ground (DGND): the digital ground was connected to the internal digital circuit of the Raspberry $\mathrm{Pi}$

(2) Analog Inputs (CH0-CH7): analog inputs 0-7 corresponded to the PPG sensor signal input pins

(3) Chip Select/Shutdown ( $\overline{\mathrm{CS}} / \mathrm{SHDN})$ : the SPI could start the communication between devices when it was set to a low voltage. The communication between the devices stopped and entered the wait state when the SPI was set to a high voltage. During communication, it must be set to a high voltage to enable communication

(4) Serial Data Output $\left(\mathrm{D}_{\mathrm{OUT}}\right)$ : when SPI data were output, the timing of the data changed because of the communication changes between devices

(5) Serial Data Input $\left(D_{I N}\right)$ : the SPI data that were input were used to upload the setting data to the communication device
(6) Serial Clock (CLK): the SPI clock pin was used to start communication

2.5. Calibration and Comparison with Standard Apparatus. To verify the accuracy of the blood pressure converted by the experimental system, this study adopted the Omron electronic blood pressure monitor as the standard apparatus for comparison (Figure 8); its specifications are presented in Table 3 [31]. In the experimental measurement, the dual PPG signals were obtained, and the blood pressure was measured simultaneously; subsequently, the dual PPG signals were extracted and converted to be used in training the BPNN. Finally, test data of the two systems were compared to determine their accuracy.

\section{Physiological Parameters Extracted from Experiments}

Physiological parameters are crucial indicators for judging the condition of the human body. The use of diverse physiological parameters can assist physicians in judging the condition of their patients. This study mainly discussed the physiological indicators related to blood pressure, including HR, PWA, PTT, PWV, and PI as the training parameters of BPNN to devise a noninvasive continuous blood pressure measurement system.

3.1. Heart Rate. The heart is an essential organ in the human body and plays a critical role in the blood circulation system. The frequency of heart beats is called HR, which is a basic 


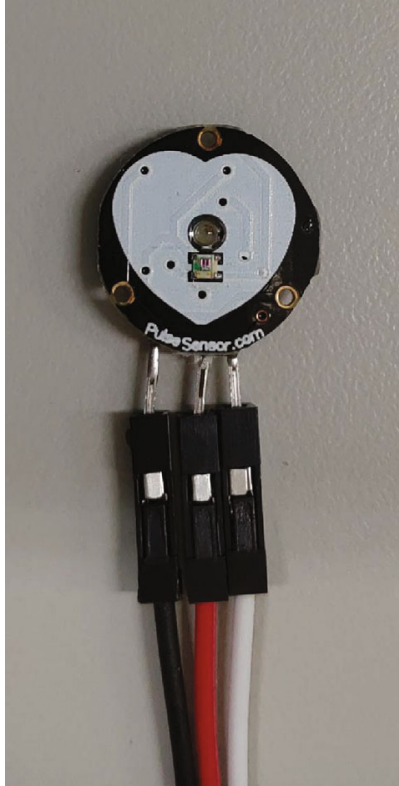

(a)

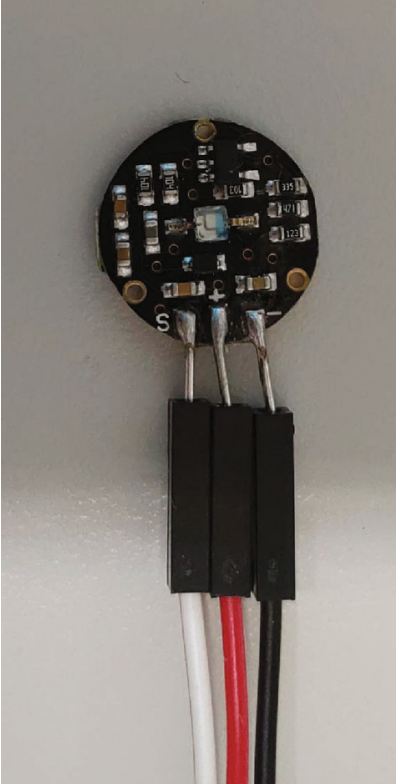

(b)

Figure 7: PPG pulse sensor: (a) front; (b) back.

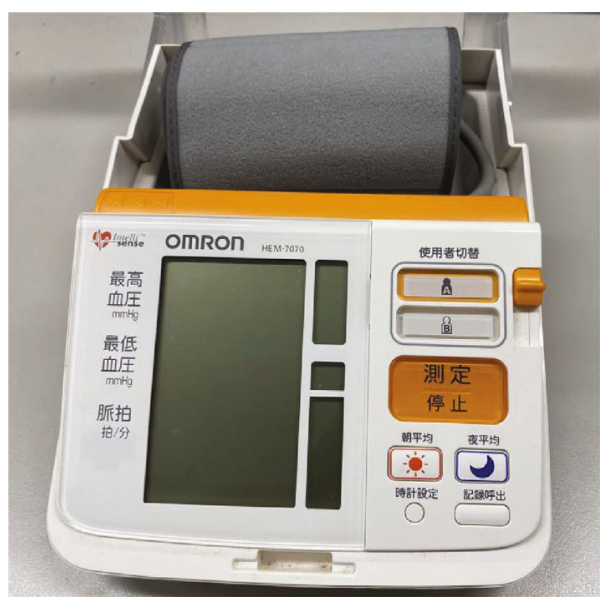

FIgURE 8: Experimental verification by Omron HEM-7000 blood pressure monitor.

indicator of the physiological condition of the human body. HR is mostly measured in patients with hypertension, coronary heart disease, heart failure, or in patients under anesthesia or in the intensive care unit. It is also one of the vital signs that clinicians use during monitoring and management. Accurate and real-time HR monitoring can serve as a reference for the clinical assessment of a patient's condition and diagnosis as well as for treatment decisions.

The most common method to estimate HR is by using ECG to extract the R peaks in the QRS complex generated during each heartbeat (the QRS complex is regarded as the criterion of a heartbeat); the heartbeat interval, called the RR interval, occurs after two successive R peaks. According to relevant literature [6], the PPG signal has the same characteristics, and it can also extract $\mathrm{P}$ waves, which exhibit the
TABLe 3: Specification of blood pressure monitor.

\begin{tabular}{lc}
\hline \multicolumn{2}{c}{ OMRON HEM-7070 electronic blood pressure monitor } \\
\hline Measurement method & Oscillometric \\
Measuring range & Pressure: $0-299 \mathrm{mmHg}$ \\
Inflation & Pulse: $40-180$ beats $/ \mathrm{min}$ \\
Deflation & Fully automatic \\
Precision & Electronic dynamic control \\
Pressure detection & Pressure: within $\pm 3 \mathrm{mmHg}$ \\
Weight & Pulse rate: within $\pm 5 \%$ read value \\
Size & Capacitive pressure sensor \\
Cuff & Approximately $990 \mathrm{~g}$ \\
& $131 \times 84 \times 155 \mathrm{~mm}(W \times H \times L)$ \\
\end{tabular}

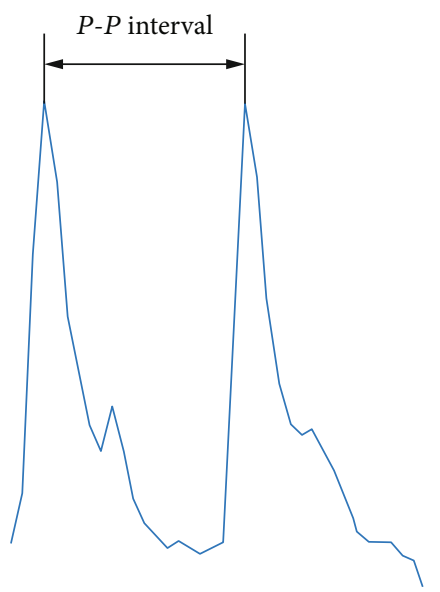

FIGURE 9: Peak-peak interval diagram. 


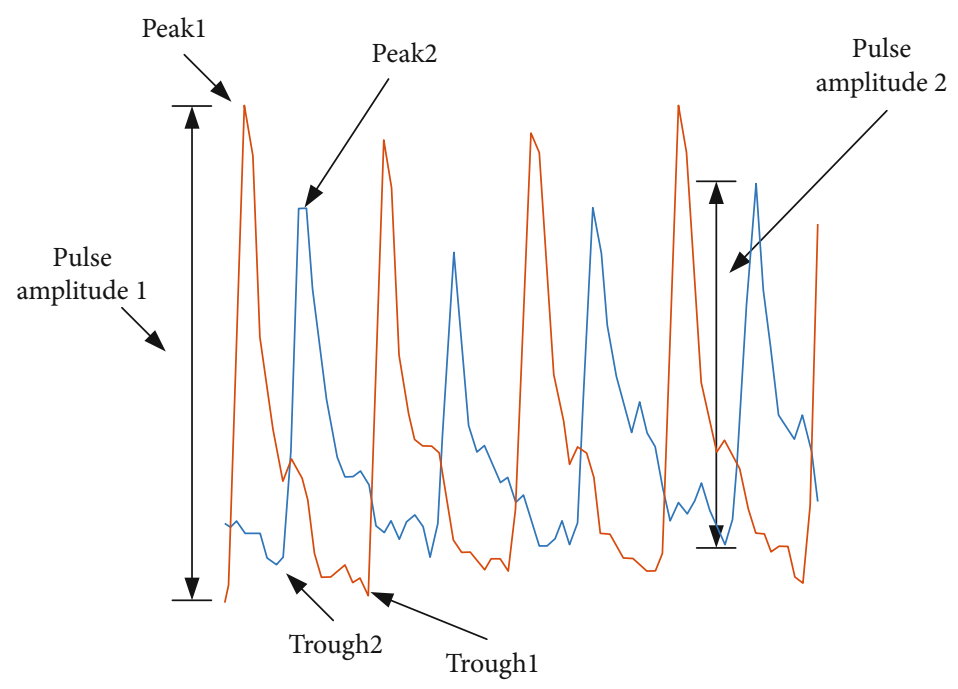

Figure 10: Pulse wave characteristic acquisition and analysis.

same characteristics as the ECG and form peak-peak intervals (Figure 9). A peak-peak interval in PPG is extracted by identifying the peak of each complete PPG signal; the time generated between each peak is the peak-peak interval. After averaging the value, it is converted to beats/min; the calculation formula is as follows:

$$
\mathrm{HR}=\left(\frac{1}{\mathrm{PPI}_{\text {mean }}}\right) \times 60 .
$$

3.2. Pulse Transit Time and Pulse Wave Velocity. PTT and PWV refer to the time and flow rate of blood in the blood vessel, respectively. The harder the vascular wall is, the faster the blood flow becomes. By contrast, softer blood vessels can absorb the effect of blood flow and, thus, reduce the flow rate. Therefore, a larger PWV indicates a harder vascular wall, which means arteriosclerosis is ongoing. According to related studies, PTT is highly correlated with the incidence of cardiovascular disease. A higher PTT suggests a higher possibility of coronary artery disease. The calculation formula of PTT is as follows:

$$
\mathrm{PWV}=\frac{D}{\mathrm{PTT}}
$$

where PTT is the pulse transit time and $D$ is the distance between the two sensors (its definition differs in other methods). According to (2), PTT must be measured before calculating PWV.

3.3. PPG Pulse Wave Analysis. An intact and undisturbed PPG pulse wave can reflect the change in blood volume when the heart beats; thus, PWA typically uses a single PPG signal to estimate blood pressure. Most related studies have extracted parameters such as systolic time, diastolic time, $1 / 2$ pulse amplitude, and maximum-minimum interval to estimate blood pressure. Although this technique improves the accuracy of blood pressure estimation, it must estimate

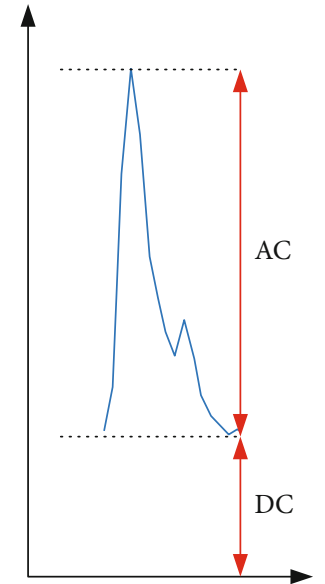

FIgure 11: Pulse AM and DC component in PPG.

blood pressure according to pulse arrival time, and that can only be achieved when a complete and stable waveform is obtained [14]. Therefore, continuous blood pressure estimation is challenging with this technique. To solve the aforementioned problem, this study simplified the waveform characteristic acquisition method by extracting stable characteristics that appear on the waveform (i.e., peak and trough values of dual PPG and arbitrary amplitude value of the dual PPG during the measurement) (as illustrated in Figure 10).

3.4. Perfusion Index. PI [32] represents the ratio of the AM to the DC component in the PPG signal. Physiologically, this index represents the change in blood volume at the measurement location, which can be used to predict the change in stroke volume caused by blood arterial circulation. Faster pulsating blood flow can result in a greater pulsating component and PI value. The PPG signal obtains the AM and DC. Physiologically, AM relates to artery compliance, which is proportional to the change in tissue blood pulsation; DC (e.g., skin and veins) is related to the smallest end-diastolic volume of blood pulsating in physiological tissues. The 


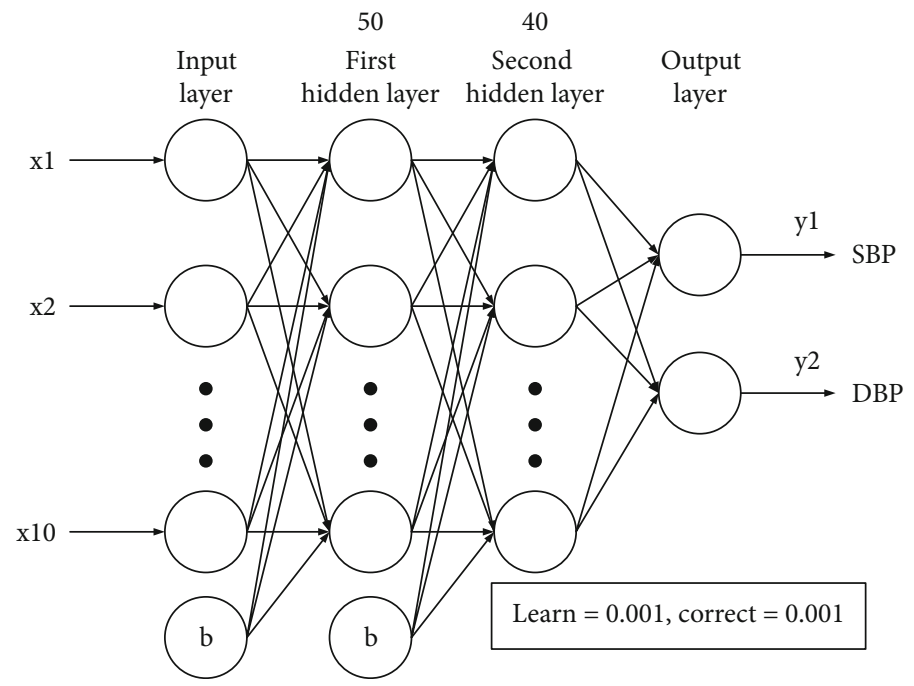

FIGURE 12: Schematic of dual PPG multifeature extraction through the use of BPNN.

PPG signal

Potentiometer

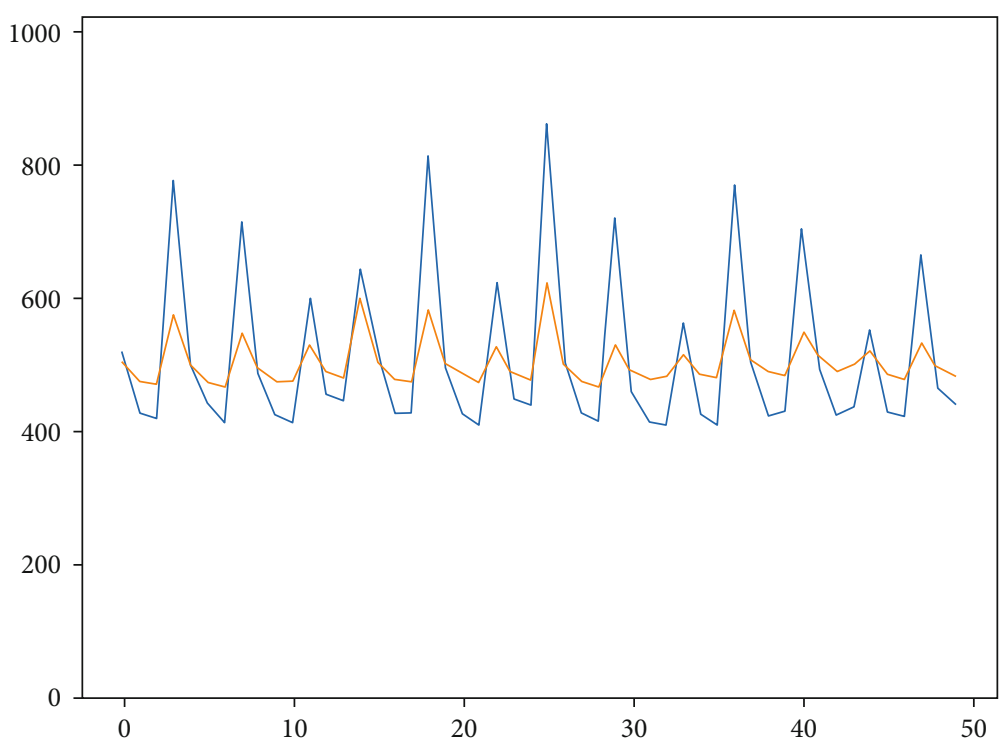

Start $-\square \times$ change in blood volume of all sections in the tissue is proportional to the DC component. The AM is obtained by subtracting the trough value $(V)$ from the peak value $(P)$, and the DC component is acquired from V (Figure 11). Therefore, the formula for PI is as follows:

$$
\mathrm{PI}=\frac{\mathrm{AM}}{\mathrm{DC}}
$$

\section{Design of the Back Propagation Neural Network}

The BPNN adopted in this experiment used the characteristics related to blood pressure on the dual PPG signals as parameters; blood pressure, measured by a standard apparatus, was added as the reference value to obtain the blood pressure optimized by the BPNN. The algorithm was classified 

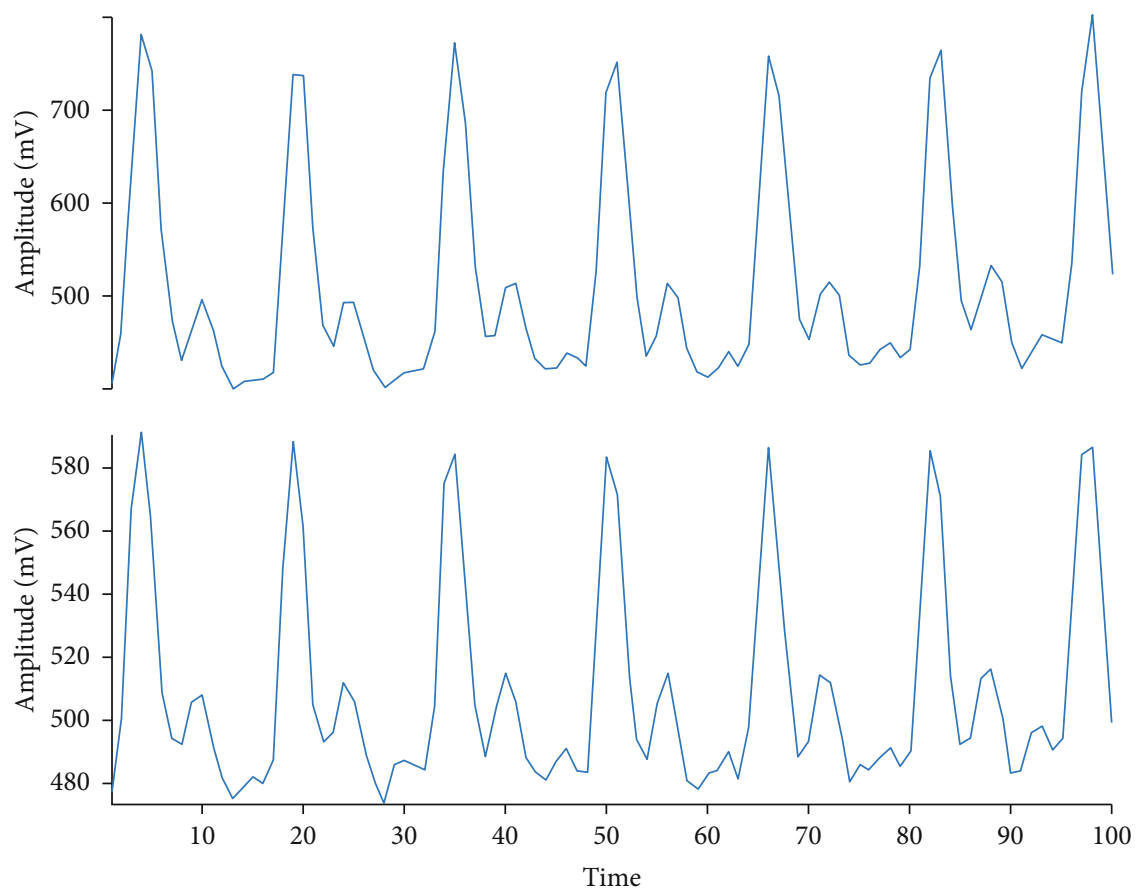

Figure 14: Measurement of dual PPG waveforms in participants without cardiovascular disease.
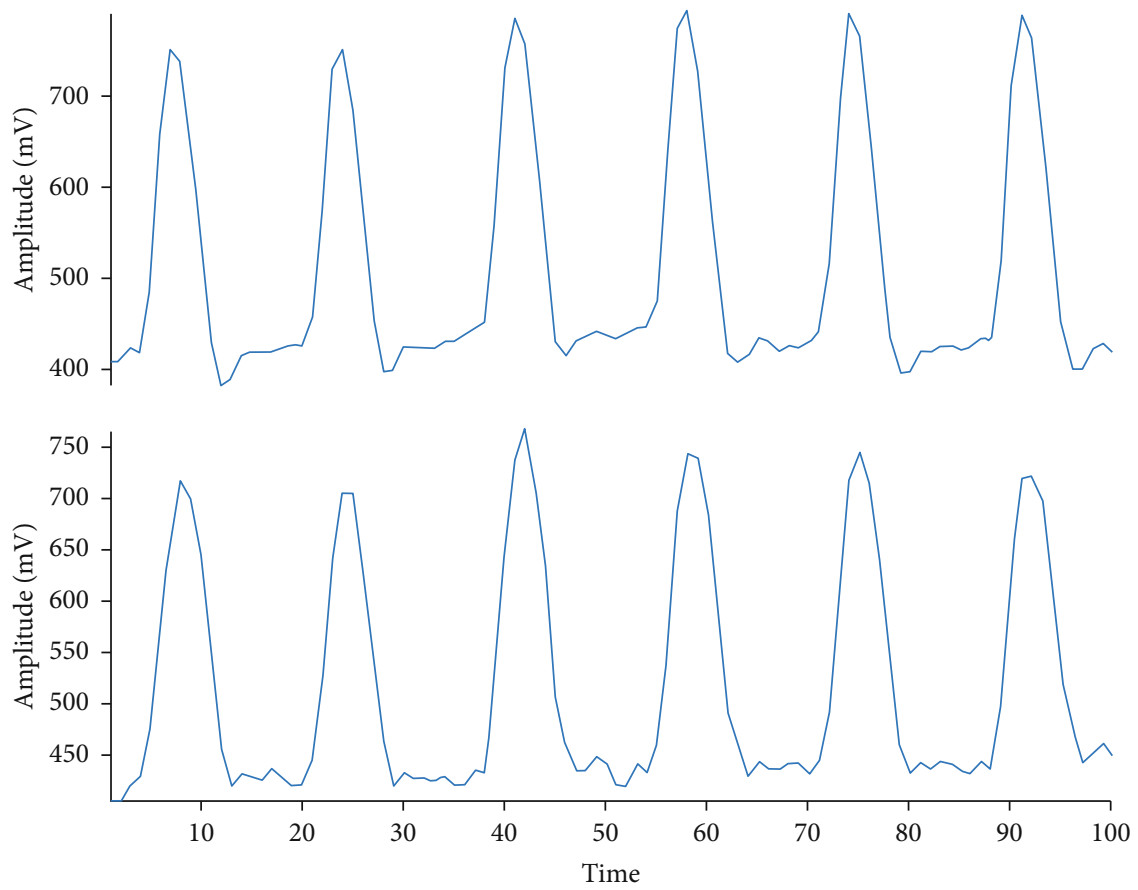

FIGURE 15: Measurement of dual PPG waveforms in participants with cardiovascular disease.

into two processes: the feedforward process and the back propagation and update process. Each iteration process forward transmitted an instance in the training set as well as calculated and saved the output of each neuron layer. The error was obtained by comparing the output of the last layer with the actual value, and the error rate of the output layer obtained from the calculation formula was used to update the weight and bias of each layer. The training sets were iteratively trained, and all the training sets were used in random order in each iteration. Finally, the predicted value was output, and the model was saved. The following network structure was designed for this experiment:

(1) Input layer

(i) Total of 10 nodes 
TABLE 4: Relationship between heart rate and blood pressure.

\begin{tabular}{lcc}
\hline HR & SBP & DBP \\
\hline 70 & 99 & 71 \\
67 & 105 & 32 \\
68 & 112 & 78 \\
58 & 125 & 68 \\
99 & 134 & 57 \\
91 & 145 & 81 \\
79 & 150 & 103 \\
76 & 168 & 114 \\
77 & 176 & 115 \\
74 & 187 & 121 \\
\hline
\end{tabular}

TABle 5: Relationship between PTT, PWV, and blood pressure.

\begin{tabular}{lccc}
\hline PTT & PWV & SBP & DBP \\
\hline 0.044 & 52.273 & 99 & 71 \\
0.043 & 53.488 & 105 & 32 \\
0.036 & 63.889 & 112 & 78 \\
0.018 & 127.778 & 125 & 68 \\
0.007 & 328.571 & 134 & 57 \\
0.007 & 328.571 & 145 & 81 \\
0.005 & 460 & 150 & 103 \\
0.003 & 657.142 & 168 & 114 \\
0.003 & 766.666 & 176 & 115 \\
0.002 & 1150 & 187 & 121 \\
\hline
\end{tabular}

TAвLE 6: Relationship between PWA and blood pressure.

\begin{tabular}{|c|c|c|c|c|c|c|c|}
\hline \multicolumn{6}{|c|}{ PWA } & \multirow{2}{*}{$\frac{\text { SBP }}{99}$} & \multirow{2}{*}{$\frac{\mathrm{DBP}}{71}$} \\
\hline 472 & 498 & 479 & 579 & 483 & 579 & & \\
\hline 487 & 508 & 477 & 533 & 490 & 551 & 105 & 32 \\
\hline 453 & 491 & 467 & 579 & 492 & 572 & 112 & 78 \\
\hline 498 & 509 & 505 & 524 & 492 & 579 & 125 & 68 \\
\hline 472 & 488 & 458 & 578 & 482 & 579 & 134 & 57 \\
\hline 493 & 497 & 451 & 516 & 478 & 579 & 145 & 81 \\
\hline 562 & 640 & 426 & 574 & 482 & 564 & 150 & 103 \\
\hline 534 & 787 & 415 & 572 & 473 & 579 & 168 & 114 \\
\hline 647 & 728 & 424 & 578 & 470 & 576 & 176 & 115 \\
\hline 559 & 844 & 384 & 579 & 463 & 578 & 187 & 121 \\
\hline
\end{tabular}

(ii) Used 10 eigenvalues extracted by PPG as the input layer

(2) First hidden layer

(i) Total of 50 nodes

(ii) Added a bias
TABLE 7: Relationship between PI and blood pressure.

\begin{tabular}{lcc}
\hline PI & SBP & DBP \\
\hline 12 & 99 & 71 \\
11 & 105 & 32 \\
12 & 112 & 78 \\
16 & 125 & 68 \\
16 & 134 & 57 \\
19 & 145 & 81 \\
15 & 150 & 103 \\
18 & 168 & 114 \\
19 & 176 & 115 \\
22 & 187 & 121 \\
\hline
\end{tabular}

(3) Second hidden layer

(i) Total of 40 nodes

(ii) Added a bias

(4) Output layer

(i) Total of two nodes

(ii) Output as SBP and DBP

The quite important issues are how to find the optimal parameters and hyperparameters of BPNN model. We first use single hidden layer BPNN model and modify the number of neurons in each layer to find the optimal solutions. If the performance is not good enough and then try to add the number of hidden layers and modify each number of neurons to decrease the errors of SBP and DBP values. After that, a total of 10 eigenvalues was input into the input layer. The activation function used to activate the first and second hidden layers was called sigmoid, and the activation function used in the process of back propagation and updating weights was called sigmoid_derivative. Adding a bias into each input layer can improve the activation of neurons. The number of neurons used in the first and second hidden layer were 50 and 40, respectively, with a learning rate of 0.001 and momentum factor of 0.001 . The output layer estimated both SBP and DBP simultaneously; the number of iterations in this study was limited to 100,000 . See Figure 12 for the network structure.

\section{Selection and Analysis of Experimental Eigenvalues}

Blood pressure is defined as the force exerted on arteries when blood enters the blood vessels. Therefore, this experiment extracted the eigenvalues from the dual PPG signals. 


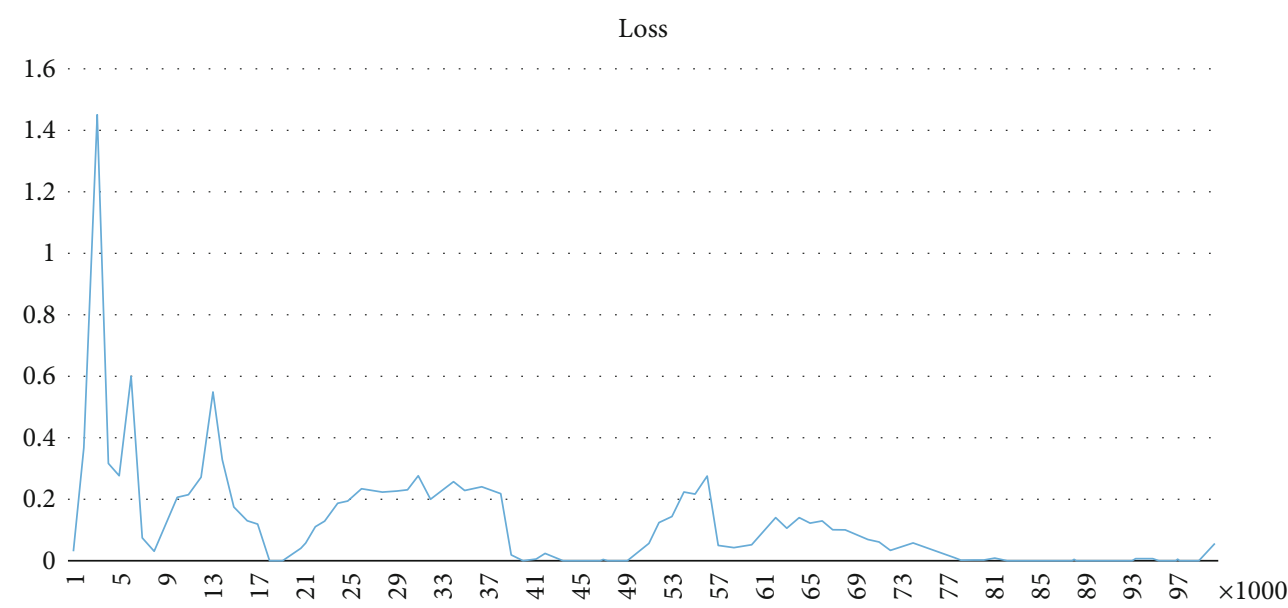

FIGURE 16: Training loss curve, recorded once every 1,000 iterations.

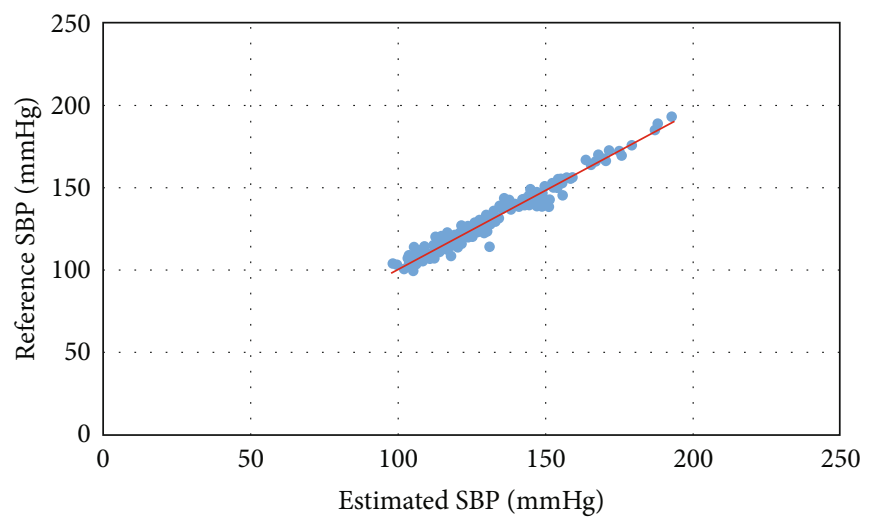

FIgURE 17: Scatter plot of the actual and estimated SBP in all participants.

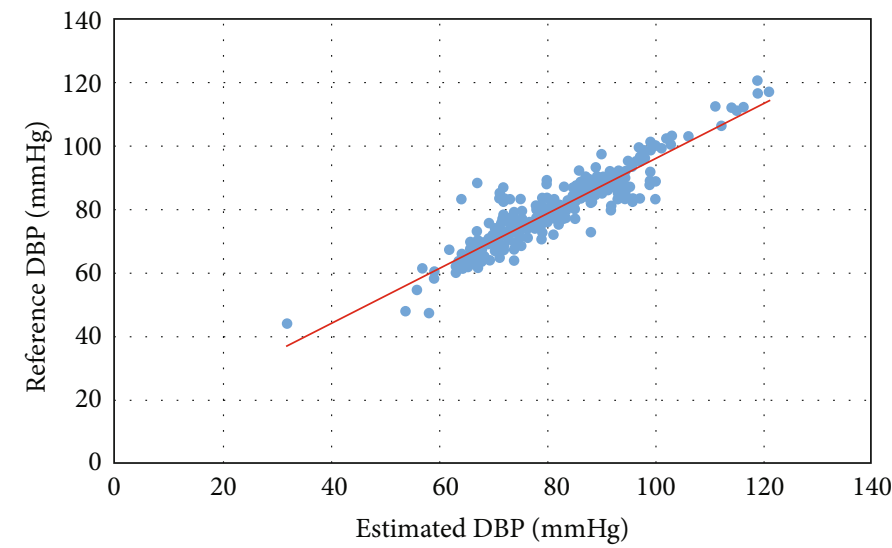

FIGURE 18: Scatter plot of the actual and estimated DBP in all participants.

The actual measurement on the graphical user interface is illustrated in Figure 13, and the dual PPG waveform measurement of healthy people and those with cardiovascular disease are shown in Figures 14 and 15, respectively. This study selected parameters that have a positive correlation with blood pressure and blood vessel condition as the characteristics to strengthen the full correlation of the neural network; these parameters were used as the inputs of the
BPNN. The experiment extracted HR, PTT, PWV, two PPG peaks, two PPG troughs, AM of the dual PPG during measurement, PI, and 10 eigenvalues. Their relationship is explained in the following sections.

5.1. HR. Under normal circumstances, people whose HR exceeds 80 beats/min have increased risk of hypertension. In 1998, a study noted that HR measurement can provide 
crucial information regarding cardiovascular risks [33]. Table 4 presents data on the relationship between HR, SBP, and DBP of the 10 participants in that experiment. The data reveal that HR increased as SBP and DBP increased.

5.2. PTT and PWV. PTT is generally defined as the time interval between the pulse wave in the aorta and the peripheral arteries, which is the peak time difference measured by two sensors simultaneously. PWV refers to the transit rate, which is calculated by dividing the distance between the two sensors by PTT. Both PTT and PWV have a strong correlation with the degree of arterial stiffness and blood pressure. Moreover, these methods have been clinically verified by medical scientists, and they have been used in medical diagnosis. Relevant studies have noted that blood pressure and PTT are inversely proportional; that is, blood pressure increases when PTT decreases [34]. Table 5 presents data on the relationship between PTT, PWV, SBP, and DBP in the 10 participants, as measured in the aforementioned experiment. As SBP and DBP increased, PTT decreased, otherwise PWV increased.

5.3. $P W A$. PWA involves PPG pulse wave analysis, which mainly retrieves all the SBP and DBP data from the PPG waveform. Some studies have analyzed related characteristic parameters, but this method mostly relies on the completeness and correctness of the waveform, which represents a challenge for real-time measurement [35]. Therefore, this experiment first used the sensor hardware to perform preliminary filtering. Subsequently, the peak and trough values of the two PPGs and the AM of the dual PPG during the measurement were extracted under a stable waveform, which represented the blood pressure-related characteristics with stable and obvious change on the PPG waveform. Table 6 presents data on the relationship between PWA, SBP, and DBP of the 10 participants measured in the aforementioned experiment. The data reveal that PWA increased as SBP and DBP increased.

5.4. PI. PI represents the change in blood volume, which can be used to predict the change in stroke volume caused by blood arterial circulation. PI refers to the ratio of pulsatile flow to nonpulsatile blood, which is also one of the essential indicators for judging the condition of blood vessels [36]. Table 7 lists data on the relationship between PI, SBP, and DBP in the 10 participants measured in the aforementioned experiment. The data reveal that PI increased as SBP and DBP increased.

\section{Experimental Results and Discussion}

The current experiment involved 100 participants. Three experimental samples were extracted from each participant, and each sample had 10 eigenvalues; thus, 300 samples were used for training the BPNN model. The 10 eigenvalues collected from the dual PPG measurement, HR, PTT, PWV, peak and trough values of dual PPGs, AM of the dual PPG during measurement, and PI were input into the BPNN for training. The results revealed that after 80,000 iterations, the error trend stabilized; the convergence curve is illustrated in Figure 16. After comparing the estimated and actual SBP and DBP, the error (mean and standard deviation) between the estimated and the actual SBP was $2.23 \pm 2.24 \mathrm{mmHg}$, the mean squared error was $3.15 \mathrm{mmHg}$, and the mean accuracy was $98.22 \%$. Moreover, the error between the estimated and the actual DBP was $3.5 \pm 3.53 \mathrm{mmHg}$, the mean squared error was $4.96 \mathrm{mmHg}$, and the mean accuracy was $95.58 \%$. The $p$ values calculated using the independent sample $t$ -test were 0.570992122 and 0.158303407 , respectively, and both were greater than 0.05 . Figures 17 and 18 reveal the scatter plots of the SBP and DBP estimated by the BPNN and the actual SBP and DBP measured by the Omron standard instrument.

\section{Conclusions}

The blood pressure measurement method proposed in this study used dual PPG sensors, which were placed on the same artery branch to simultaneously capture pulses from fingers. The signals from both sensors were synchronized, and the waveforms were recorded in real time. Moreover, cardiovascular disease-related indicators such as the peak and trough values of PPG pulse, HR, PI, PTT, and PWV were used in the BPNN algorithm to estimate the SBP and DBP. The BPNN was designed with robust experimental data and integrated with blood pressure-related indicators; this enabled the researchers to effectively train the model and complete the continuous blood pressure measurement system. Moreover, to increase the robustness of the model, this study input data of 100 Asian participants (including those with and without cardiovascular disease, each with a proportion of approximately 50\%) into the BPNN training database. The designed system only requires 10 eigenvalues to instantly output blood pressure values. In the proposed real-time blood pressure measurement system, the mean accuracy of SBP and DBP was $98.22 \%$ and $95.58 \%$, respectively.

\section{Data Availability}

Our experiment data restrict by computer-processed personal data protection law in Taiwan.

\section{Conflicts of Interest}

The authors declare that they have no conflicts of interest.

\section{Acknowledgments}

This study was supported in part by the Ministry of Science and Technology MOST 108-2221-E-150-022-MY3.

\section{References}

[1] A. B. Hertzman and C. R. Spielman, "Observations on the finger volume pulse recorded photoelectrically," The American Journal of Physiology, vol. 119, pp. 334-335, 1937.

[2] A. C. Burton, "The range and variability of the blood flow in the human fingers and the vasomotor regulation by body temperature," American Physiological Society, vol. 127, pp. 437453, 1939. 
[3] Y. Mendelson and B. D. Ochs, "Noninvasive pulse oximetry utilizing skin reflectance photoplethysmography," IEEE Transactions on Biomedical Engineering, vol. 35, no. 10, pp. 798-805, 1988.

[4] M. Nitzan, H. de Boer, S. Turivnenko, A. Babchenko, and D. Sapoznikov, "Power spectrum analysis of spontaneous fluctuations in the photoplethysmographic signal," Journal of basic and clinical physiology and pharmacology, vol. 5, no. 34, pp. 269-276, 1994.

[5] M. Marek, J. Thomas Bigger, A. John Camm et al., "Heart rate variability: standards of measurement, physiological interpretation, and clinical use," European Heart Journal, vol. 17, no. 3, pp. 354-381, 1996.

[6] K. Nakajima, T. Tamura, and H. Miike, "Monitoring of heart and respiratory rates by photoplethysmography using a digital filtering technique," Medical Engineering \& Physics, vol. 18, no. 5, pp. 365-372, 1996.

[7] S. Rhee, B.-H. Yang, and H. H. Asada, "Artifact-resistant power-efficient design of finger-ring plethysmographic sensors," IEEE Transactions on Biomedical Engineering, vol. 48, no. 7, pp. 795-805, 2001.

[8] K. W. Chan, K. Hung, and Y. T. Zhang, "Noninvasive and cuffless measurements of blood pressure for telemedicine," in 2001 Conference Proceedings of the 23rd Annual International Conference of the IEEE Engineering in Medicine and Biology Society, vol. 4, pp. 3592-3593, Istanbul, Turkey, 2001.

[9] V. S. Murthy, S. Ramamoorthy, N. Srinivasan, S. Rajagopal, and M. M. Rao, "Analysis of photoplethysmographic signals of cardiovascular patients," in 2001 Conference Proceedings of the 23rd Annual International Conference of the IEEE Engineering in Medicine and Biology Society, vol. 3, pp. 22042207, Istanbul, Turkey, 2001.

[10] Y. Y. Gu, Y. Zhang, and Y. T. Zhang, "A novel biometric approach in human verification by photoplethysmographic signals," in 4th International IEEE EMBS Special Topic Conference on Information Technology Applications in Biomedicine, pp. 13-14, Birmingham, UK, 2003.

[11] J. Liu, Y. Zhang, X. Ding, W. Dai, and N. Zhao, "A preliminary study on multi-wavelength PPG based pulse transit time detection for cuffless blood pressure measurement," in 2016 38th Annual International Conference of the IEEE Engineering in Medicine and Biology Society (EMBC), pp. 615-618, Orlando, FL, 2016.

[12] H. Lui and K. Chow, "A novel calibration procedure of pulse transit time based blood pressure measurement with heart rate and respiratory rate," in 2018 40th Annual International Conference of the IEEE Engineering in Medicineand Biology Society (EMBC), pp. 4318-4322, Honolulu, HI, 2018.

[13] D. Czerwinski, P. Wojcicki, and T. Zientarski, "On time blood pressure prediction with the use of PPG signals," in 2017 International Conference on Electromagnetic Devices and Processes in Environment Protection with Seminar Applications of Superconductors (ELMECO \& AoS), pp. 1-4, Lublin, 2017.

[14] A. Savkar, P. Khatate, and C. Y. Patil, "Study on techniques involved in tourniqueteless blood pressure measurement using PPG," in 2018 Second International Conference on Intelligent Computing and Control Systems (ICICCS), pp. 170-172, Madurai, India, 2018.

[15] R. Shriram, A. Wakankar, N. Daimiwal, and D. Ramdasi, "Continuous cufflessblood pressure monitoring based on PTT," in 2010 International Conference on Bioinformatics and Biomedical Technology, pp. 51-55, Chengdu, 2010.
[16] M. H. Pollak and P. A. Obrist, "Aortic-radial pulse transit time and ECG Q-wave to radial pulse wave interval as indices of beat-by-beat blood pressure change," Psychophysiology, vol. 20, no. 1, pp. 21-28, 1983.

[17] L. A. Geddes, M. H. Voelz, C. F. Babbs, J. D. Bourland, and W. A. Tacker, "Pulse transit time as an indicator of arterial blood pressure," Psychophysiology, vol. 18, no. 1, pp. 71-74, 1981.

[18] A. A. Robert, A. S. John, M. D. Dennis, A. W. Mark, and C. B. Taylor, "The covariation of blood pressure and pulse transit time in hypertensive patients," Psychophysiology, vol. 18, pp. 301-306, 1981.

[19] G. V. Marie, C. R. Lo, J. Van Jones, and D. W. Johnston, "The relationship between arterial blood pressure and pulse transit time during dynamic and static exercise," Psychophysiology, vol. 21, no. 5, pp. 521-527, 1984.

[20] J. Kerola, V. Kontra, and R. Sepponen, "Non-invasive blood pressure data acquisition employing pulse transit lime detection," in Proceedings of the 18th Annual International Conference of the IEEE Medicine and Biology Society, vol. 3, pp. 1308-1309, Amsterdam, Netherlands, 1996.

[21] K. Meigas, R. Kattai, and J. Lass, "Continuous blood pressure monitoring using pulse wave delay," in Proceedings of the $23 \mathrm{rd}$ Annual International Conference of the IEEE Medicine and Biology Society, vol. 4, pp. 3171-3174, Istanbul, Turkey, 2001.

[22] A. Rasool, M. Rafiq, A. Nasir, and F. M. Kashif, "Continuous and noninvasive blood pressure estimation by two-sensor measurement of pulse transit time," in 2018 14th International Conference on Emerging Technologies (ICET), pp. 1-5, Islamabad, 2018.

[23] W. Lin, H. Wang, O. W. Samuel, and G. Li, "Using a new PPG indicator to increase the accuracy of PTT-based continuous cuffless blood pressure estimation," in 2017 39th Annual International Conference of the IEEE Engineering in Medicine and Biology Society (EMBC), pp. 738-741, Seogwipo, 2017.

[24] R. Nye, Z. Zhang, and Q. Fang, "Continuous non-invasive blood pressure monitoring using photoplethysmography: a review," in 2015 International Symposium on Bioelectronics and Bioinformatics (ISBB), pp. 176-179, Beijing, 2015.

[25] L. Peter, N. Noury, and M. Cerny, "A review of methods for non-invasive and continuous blood pressure monitoring: Pulse transit time method is promising?," Irbm, vol. 35, no. 5, pp. 271-282, 2014.

[26] D. B. McCombie, A. T. Reisner, and H. H. Asada, "Adaptive blood pressure estimation from wearable PPG sensors using peripheral artery pulse wave velocity measurements and multichannel blind identification of local arterial dynamics," in Annual International Conference of the IEEE Engineering in Medicine and Biology-Proceedings, pp. 3521-3524, New York, NY, USA, 2006.

[27] R. Samria, R. Jain, A. Jha, S. Saini, and S. R. Chowdhury, "Noninvasive cuffless estimation of blood pressure using photoplethysmography without electrocardiograph measurement," in IEEE TENSYMP 2014 -2014 IEEE Region 10 Symposium, pp. 254-257, Kuala Lumpur, Malaysia, 2014.

[28] G. Joseph, A. Joseph, G. Titus, R. M. Thomas, and D. Jose, "Photoplethysmogram (PPG) signal analysis and wavelet de-noising," in 2014 Annual International Conference on Emerging Research Areas: Magnetics, Machines and Drives (AICERA/iCMMD), pp. 1-5, Kottayam, 2014.

[29] C. Fischer, B. Dömer, T. Wibmer, and T. Penzel, "An algorithm for real-time pulse waveform segmentation and artifact 
detection in photoplethysmograms," IEEE Journal of Biomedical and Health Informatics, vol. 21, no. 2, pp. 372-381, 2017.

[30] Y. Zhang and Z. Wang, “A hybrid model for blood pressure prediction from a PPG signal based on MIV and GA-BP neural network," in 2017 13th International Conference on Natural Computation, Fuzzy Systems and Knowledge Discovery (ICNCFSKD), pp. 1989-1993, Guilin, 2017.

[31] P. Boutouyrie, M. Briet, C. Collin, S. Vermeersch, and B. Pannie, "Assessment of pulse wave velocity," Artery Research, vol. 3, pp. 3-8, 2009.

[32] W. Mower, G. Myers, E. Nicklin, K. Kearin, L. Baraff, and C. Sachs, "Pulse oximetry as a fifth vital sign in emergency geriatric assessment," Academic Emergency Medicine, vol. 5, no. 9, pp. 858-865, 1998.

[33] G. A. Lanza, V. Guido, M. M. Galeazzi et al., "Prognostic role of heart rate variability in patients with a recent acute myocardial infarction," The American Journal of Cardiology, vol. 82, no. 11, pp. 1323-1328, 1998.

[34] B. Mishra and N. Thakkar, "Cuffless blood pressure monitoring using PTT and PWV methods," in 2017 International Conference on Recent Innovations in Signal processing and Embedded Systems (RISE), pp. 395-401, Bhopal, 2017.

[35] Y. Kurylyak, F. Lamonaca, and D. Grimaldi, "A neural network-based method for continuous blood pressure estimation from a PPG signal," in 2013 IEEE International Instrumentation and Measurement Technology Conference (I2MTC), pp. 280-283, Minneapolis, MN, 2013.

[36] T. Y. Abay and P. A. Kyriacou, "Reflectance photoplethysmography as noninvasive monitoring of tissue blood perfusion," IEEE Transactions on Biomedical Engineering, vol. 62, no. 9, pp. 2187-2195, 2015. 